\title{
Dispatching of Wind/Battery Energy Storage Hybrid Systems Using Inner Point Method-Based Model Predictive Control
}

\author{
Deyou Yang ${ }^{1}$, Jiaxin Wen ${ }^{1, *}$, Ka-wing Chan ${ }^{2}$ and Guowei Cai ${ }^{1}$ \\ 1 School of Electrical Engineering, Northeast Dianli University, Jilin 132012, China; \\ eedyyang@hotmail.com (D.Y.); caigw@nedu.edu.cn (G.C.) \\ 2 Department of Electrical Engineering, Hong Kong Polytechnic University, Hong Kong, China; \\ eekwchan@polyu.edu.hk \\ * Correspondence: jiaxinwen72@outlook.com; Tel.: +86-432-6480-6691 \\ Academic Editors: Shuhui Li, Eduardo Alonso and Xingang Fu \\ Received: 9 July 2016; Accepted: 8 August 2016; Published: 11 August 2016
}

\begin{abstract}
The application of large scale energy storage makes wind farms more dispatchable, which lowers operating risks to the grid from interconnected large scale wind farms. In order to make full use of the flexibility and controllability of energy storage to improve the schedulability of wind farms, this paper presents a rolling and dispatching control strategy with a battery energy storage system (BESS) based on model predictive control (MPC). The proposed control scheme firstly plans expected output, i.e., dispatching order, of a wind/battery energy storage hybrid system based on the predicted output of the wind farm, then calculates the order in the predictive horizon with the receding horizon optimization and the limitations of energy storage such as state of charge and depth of charge/discharge to maintain the combination of active output of the wind farm and the BESS to track dispatching order at the extreme. The paper shows and analyses the effectiveness of the proposed strategy with different sizes of capacity of the BESS based on the actual output of a certain actual wind farm in the northeast of China. The results show that the proposed strategy that controls the BESS could improve the schedulability of the wind farm and maintain smooth output of wind/battery energy storage hybrid system while tracking the dispatching orders. When the capacity of the BESS is $20 \%$ or the rated capacity of the wind farm, the mean dispatching error is only $0.153 \%$ of the rated capacity of the wind farm.
\end{abstract}

Keywords: battery energy storage; wind power; combination active output; dispatching curve

\section{Introduction}

Wind power technology is the most mature and widespread applied one among new energy power generation technologies. Exploration of wind energy on a large scale could substantialy alleviate the problems of energy shortages and environmental pollution across the globe. Up to the end of 2015, the global installed wind capacity had reached $432.4 \mathrm{GW}$, of which the figure in China was $148 \mathrm{GW}$, representing the largest share around the world [1]. Random output of wind speed lowers the schedulability of active power in a wind farm, which in turn limits the availability of wind energy [2]. With increasing permeability of wind power in the state grid, lower schedulability would increase operational risks, especially wind power curtailment [3,4].

Proven large scale battery energy storage system (BESS) technology and its application in the grid provides a new platform to increase the utilization rate of new energy sources [5-9], to improve the schedulability in a wind farm and to guarantee the availability to the grid of high wind power that can operate safely and steadily $[10,11]$. In recent years, domestic and foreign scholars have studied control strategies for BESS that have the ability of charging and discharging power rapidly to smooth 
fluctuating wind power widely and deeply [12-20]. So far the most widely applied control algorithm is low pass filtering (LPF) that obtains the wind power target after the actual wind power is sent to the first order filter and then treats the difference between the target and actual wind power as a compensating instruction sent to the BESS. In $[16,17]$, the authors proposed a moving average control algorithm which control principle is similar to that of LPF. In [18], the authors decomposed the wind power with the wavelet transform (WT), and then hybrid energy storage was applied to smooth the corresponding fluctuating component at different frequencies, which alleviated the pressure of fluctuating wind power after the wind plant was integrated into the grid. In [19], the authors proposed a dynamic programming (DP) algorithm with the consideration of state of charge (SOC) of the BESS to smooth fluctuating wind power. In [20], the authors obtained a smoothing target which minimized the action of the BESS, and then Model Predictive Control (MPC) was applied to make the total output smooth.

To sum up, the common objective of the above control algorithms is to smooth the output of wind farms and reduce the deviation between the target and actual wind power to the greatest extent, but no dispatch order is introduced, which limits the contribution of these methods to the schedulability of the wind plant. MPC is wildly applied with good results in the fields of renewable energy generation and control. In [21], current control of a Hybrid Fuel Cell Power system is realized. In [22], frequency/voltage control of a wind-diesel power system based on MPC is presented. In [23], MPC is applied in dispatching active wind power of an off-grid wind turbine/photovoltaic/hydrogen/battery hybrid system. In [24], active power control for a wind/electrolyzer/fuel cell hybrid system based on non-linear MPC is proposed. This paper considers the grid requirements and proposes the MPC for the BESS to improve the schedulability of the wind farm, and then compares the effects of two methods of solving for MPC, the Inner Point (IP) method (IP-MPC) and the Quadratic Programming (QP) method (QP-MPC). Firstly, the wind power dispatch order in the coming dispatching period (30 min) is obtained according to the predictive wind power, and this is regarded as the operational target for the wind/battery energy storage hybrid system, then the control command for the BESS in the dispatching period is acquired through MPC, which can ensure the total output of the wind/battery energy storage hybrid system could be injected into the grid smoothly and safely.

The remainder of the paper is organized as follows. Firstly, the dynamic model of the wind/battery energy storage hybrid system is introduced in Section 2. Second, the dispatching strategy for a wind/battery energy storage hybrid system based on MPC is proposed in Section 3 . Then the presented dispatching strategy is investigated by application to a certain wind farm in the northeast of China in Section 4 .

\section{The Wind/Battery Energy Storage Hybrid System Modeling}

\subsection{System Configuration}

The overall structure of the wind/battery energy storage hybrid system is illustrated in Figure 1. The system is consist of a wind farm, a BESS, a converter and a power management unit (PMU), and the system is coupled to the main grid via transmission line. The BESS is connected to the grid at the point of common coupling (PCC) and charges/discharges power via the converter to smooth the wind power injected into the grid.

This paper aims at improving the schedulability of the wind farm, which means to enable the output of the wind/battery storage hybrid system to approach the desired dispatch curve $P_{\mathrm{D}}$ as much as possible. In order to achieve the goal, a wind power forecasting model is applied to predict future $p$-step wind power at each sampling point, and the dispatch order is based on these predicted values. The reasonable action of the BESS $\left(P_{\mathrm{B}}\right)$ is calculated by the control strategy in the PMU to compensate for the difference between the dispatch order $P_{\mathrm{D}}$ and the actual wind power $P_{\mathrm{W}}$. Under this strategy the power injected to the grid (total output of the wind/battery energy storage hybrid system) $P_{\mathrm{G}}$ 
would follow the dispatch order from the transmission system operator (TSO), and the effects mainly depends on the capacity of the battery and the control algorithm in the PMU.

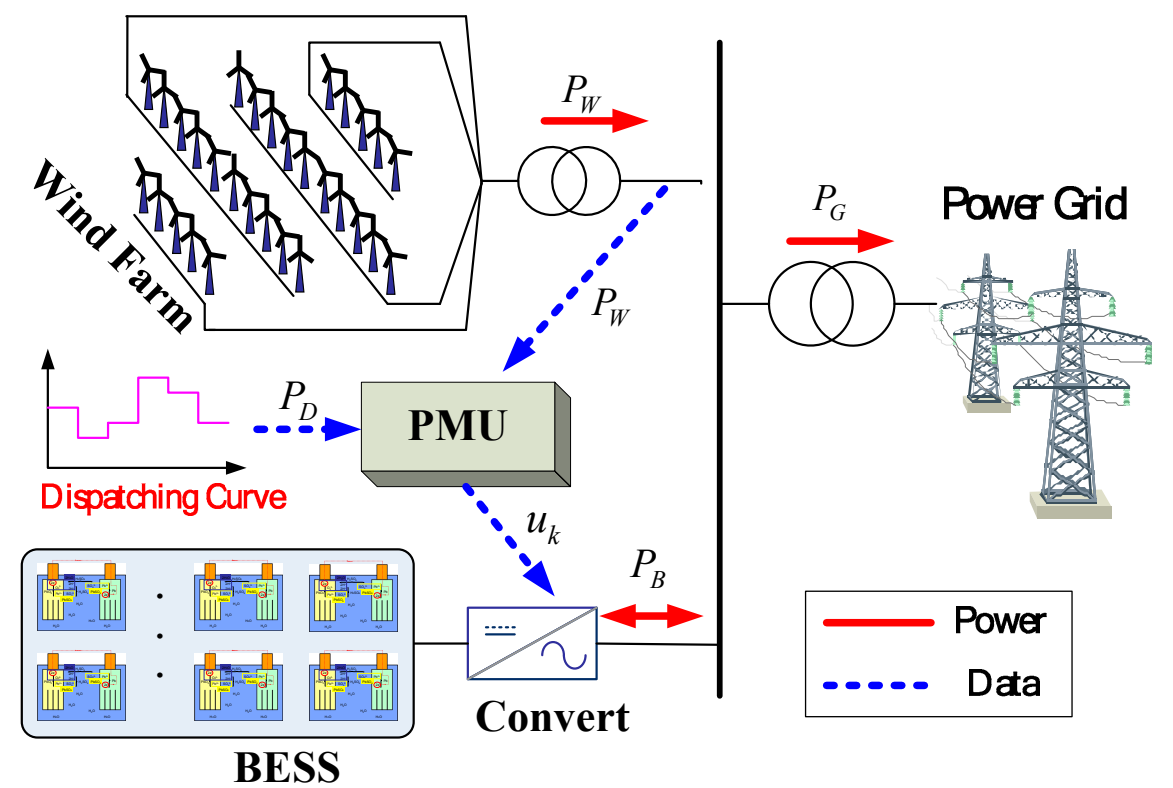

Figure 1. Structure chart of wind/battery energy storage hybrid system.

\subsection{System Modeling}

According to Figure 1 and the analysis in Section 2.1, the model of wind/battery energy storage hybrid system can be formulated as [25]:

$$
\begin{gathered}
P_{\mathrm{G}}(k+1)=P_{\mathrm{B}}(k)+P_{\mathrm{W}}(k) \\
E_{\mathrm{B}}(k+1)=E_{\mathrm{B}}(k)-\Delta T_{\mathrm{B}} P_{\mathrm{B}}(k)
\end{gathered}
$$

where $P_{\mathrm{G}}(k)$ is the total output of the wind/battery energy storage hybrid system; $P_{\mathrm{B}}(k)$ is the power control signal for the BESS. When $P_{\mathrm{B}}(k)>0$, it denotes the BESS discharges power; when $P_{\mathrm{B}}(k)<0$, it denotes the BESS charges power. $P_{\mathrm{W}}(k)$ denotes the actual wind power, $E_{\mathrm{B}}(k)$ denotes the remaining energy in the BESS. State of Charge (SOC) of a BESS is the ratio between available energy and its rated capacity, expressed as a percentage. $\Delta T_{\mathrm{B}}$ is the transfer coefficient from MW to MWh, for instance when the sampling time is $5 \mathrm{~min}, \Delta T_{\mathrm{B}}=5 \mathrm{~min} / 60 \mathrm{~min}=1 / 12$.

Equation (1) can be written in the form of state space as follows:

$$
\begin{aligned}
& x(k+1)=\mathbf{A} x(k)+\mathbf{B}_{1} u(k)+\mathbf{B}_{2} d(k) \\
& y(k)=\mathbf{C} x(k)
\end{aligned}
$$

with, state vector:

$$
x(k)=\left[\begin{array}{c}
x_{1}(k) \\
x_{2}(k)
\end{array}\right]=\left[\begin{array}{c}
P_{\mathrm{G}}(k) \\
E_{\mathrm{B}}(k)
\end{array}\right]
$$

control input: $u(k)=P_{\mathrm{B}}(k)$; system output: $y(k)=P_{\mathrm{G}}(k)$; combined with the system:

$$
\begin{aligned}
\mathbf{A}=\left[\begin{array}{ll}
0 & 0 \\
0 & 1
\end{array}\right], \quad \mathbf{C}=\left[\begin{array}{ll}
1 & 0
\end{array}\right] \\
\mathbf{B}_{1}=\left[\begin{array}{c}
1 \\
-\Delta T_{\mathbf{B}}
\end{array}\right], \quad \mathbf{B}_{2}=\left[\begin{array}{l}
1 \\
0
\end{array}\right]
\end{aligned}
$$


The controllability matrix $Q=\left[\mathrm{B}_{1}, \mathrm{AB}_{1}\right]$ is full rank, so the system is controllable.

With consideration of the technology and economy, in actual operation the BESS is limited by the SOC and charging/discharging power:

\section{(A) SOC Constraint}

The lifetime of a BESS is influenced by the depth of discharge (DoD), and in order to prolong the lifespan of a BESS and reduce costs, there would be a strict constraint on SOC of a BESS at any time $k$ :

$$
S_{\min } \leqslant x_{2}(k) / C_{\mathrm{B}} \leqslant S_{\max }, k=1,2, \cdots, P
$$

where, $x_{2}(k)$ denotes the remaining energy at time $k ; C_{\mathrm{B}}$ denotes rate capacity of a BESS; $S_{\text {min }}$ denotes lower limit of a BESS, $S_{\max }$ denotes upper limit of a BESS.

\section{(B) Charge/Discharge Power Constraint}

To ensure the BESS can operate safely, there would be strict constraints on the value of charging/discharging power in the actual BESS system:

$$
\begin{gathered}
P_{c}=\min \left(\left(S_{\max }-S(k-1)\right) C_{\mathrm{B}} / \Delta T_{\mathrm{B}}, P_{\text {max } \_ \text {ch }}\right) \\
P_{d}=\min \left(\left(S(k-1)-S_{\min }\right) C_{\mathrm{B}} / \Delta T_{\mathrm{B}}, P_{\text {max } \_\mathrm{f}}\right)
\end{gathered}
$$

where, $P_{c}(k)$ and $P_{d}(k)$ are the available charging power and discharging power of the BESS, respectively, $P_{\text {max_ch }}$ and $P_{\text {max_f }}$ are the maximum charging power and discharging power separately.

\section{Dispatching Strategy for Wind/Battery Energy Storage Hybrid System Based on MPC}

\subsection{Wind Power Predicting and Dispatch Curve}

This paper aims at improving the schedulability for the wind farm using BESS, i.e., the total active output of the wind/battery energy storage hybrid system $\left(P_{\mathrm{G}}\right)$ could trace the dispatch curve. Therefore, a reasonable dispatch order is significant to achieve the goal.

The paper focuses on calculating action order of the BESS, not the method on predicting wind power, therefore the method called ARIMA is adapted to predict wind power [26]. The model of ARMA could expressed as:

$$
\mathrm{z}_{t}=\varphi_{1} z_{t-1}+\cdots+\varphi_{p} z_{t-p}+a_{t}-\theta_{1} a_{t-1}-\cdots-\theta_{q} a_{t-q}
$$

where, $\varphi_{1}, \varphi_{2}, \ldots, \varphi_{p}$ and $\theta_{1}, \theta_{2}, \ldots, \theta_{q}$ are all constants. $a_{t}$ is white noise time series, therefore time series $\left\{z_{t}\right\}$ comply with ARMA with the order of $(p, q)$, described as $\operatorname{ARMA}(p, q)$. Wind power is a non-stationary time series that needs to be tackled with difference processing. After being processed $\mathrm{d}$ times, $\left\{\nabla^{d} z_{t}\right\}$ becomes a stationary ARMA series, therefore:

$$
\varphi_{p}(F) \nabla^{d} z_{t}=\theta_{q}(F) a_{t}
$$

It is ARMA with the order of $(p, d, q)$, described as $\operatorname{ARIMA}(p, d, q), \mathrm{F}$ is the backward difference operator. The key for predicting wind power with ARMA is to identify the parameters of $\varphi_{1}, \varphi_{2}, \varphi_{p}$ and $\theta_{1}, \theta_{2}, \theta_{q}$. In the paper, least squares estimation is used to identify the parameters which involves minimizing the residual sum of squares.

$$
\sum_{i=1}^{N} a_{t}^{2}=\sum_{i=1}^{N}\left(\theta_{q}^{-1}(F) \varphi_{p}(F) \nabla^{d} z_{t}\right)^{2}
$$


With the consideration of computing time and computing accuracy, in the paper the data in the last $12 \mathrm{~h}$ with 144 wind power data is sampled to forecast future wind power in $0.5 \mathrm{~h}$ with six wind power data.

The dispatch order is made based on wind power forecasting values, as a consequence the precision of prediction in a sense could impact the control effects. The rules that all the generation units submit the dispatch power that the plants have to send into the grid to the TSO in each dispatching interval [9] must be satisfied. Therefore, the dispatch power is designed to meet a constant value in each dispatch interval and is obtained by averaging the predictive wind power in each dispatch interval. In the $k^{\text {th }}$ dispatching interval i.e., $k T_{d}<t \leqslant(k+1) T_{d}$, the dispatch power is defined as follows:

$$
P_{\mathrm{D}}=\frac{1}{T_{d}} \int_{k T_{d}}^{(k+1) T_{d}} P_{\mathrm{w}}(\tau) d \tau
$$

where, $T_{d}$ is the dispatching interval and it is assumed to be $30 \mathrm{~min}$ in this paper. The control interval of the BESS is $5 \mathrm{~min}$. The sampling time interval of actual wind power is $5 \mathrm{~min}$ and the interval of predictive wind power is also $5 \mathrm{~min}$. The method of acquiring the dispatch order is illustrated in Figure 2.

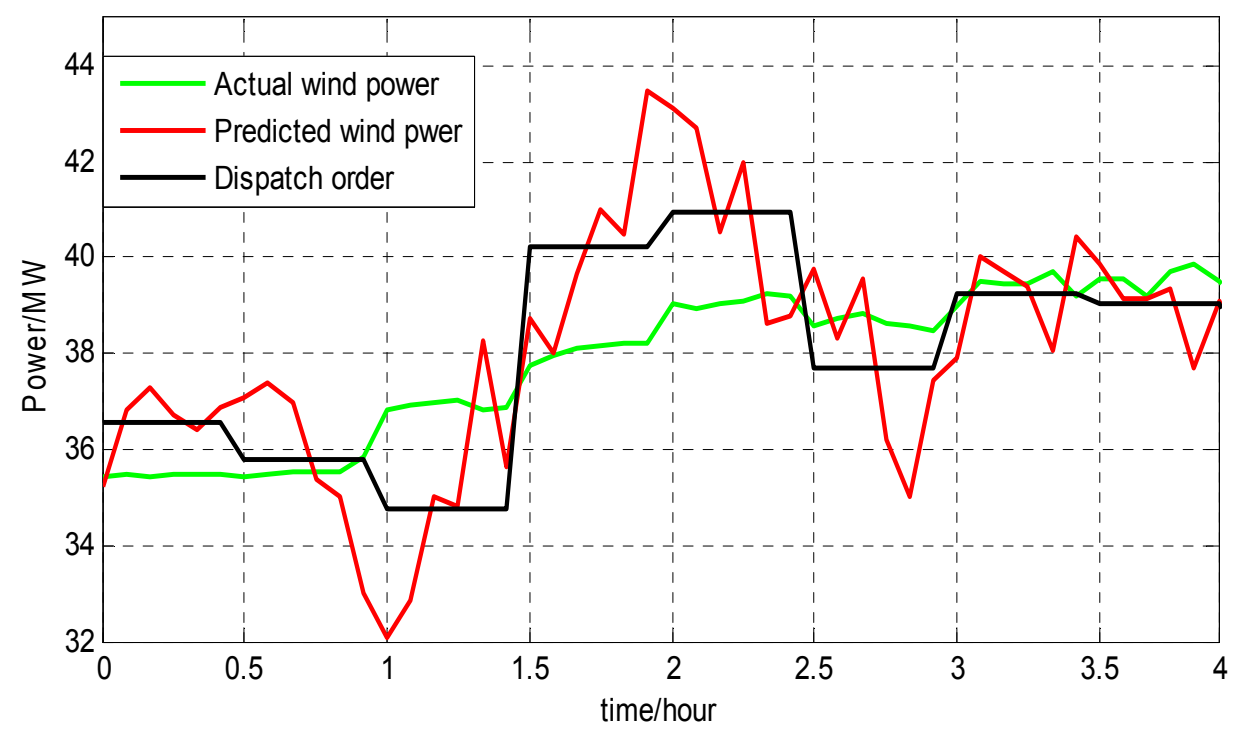

Figure 2. Schematic diagram of selecting dispatching order.

At the beginning of each dispatching interval, the dispatch order is obtained by averaging the predicted wind power and is selected as the target for MPC in this paper to enable the total output of the system to track correctly.

\subsection{Design for MPC Contoller for Dispatching}

Model Predictive Control (MPC) is a method to solve online optimal control problems. It can find out present and a series of future control values according to the state $x(k)$ and present and a series of future constraints. The control strategy for MPC proposed in the paper is illustrated in Figure 3 and it is comprised of a predictive model and rolling optimization. 


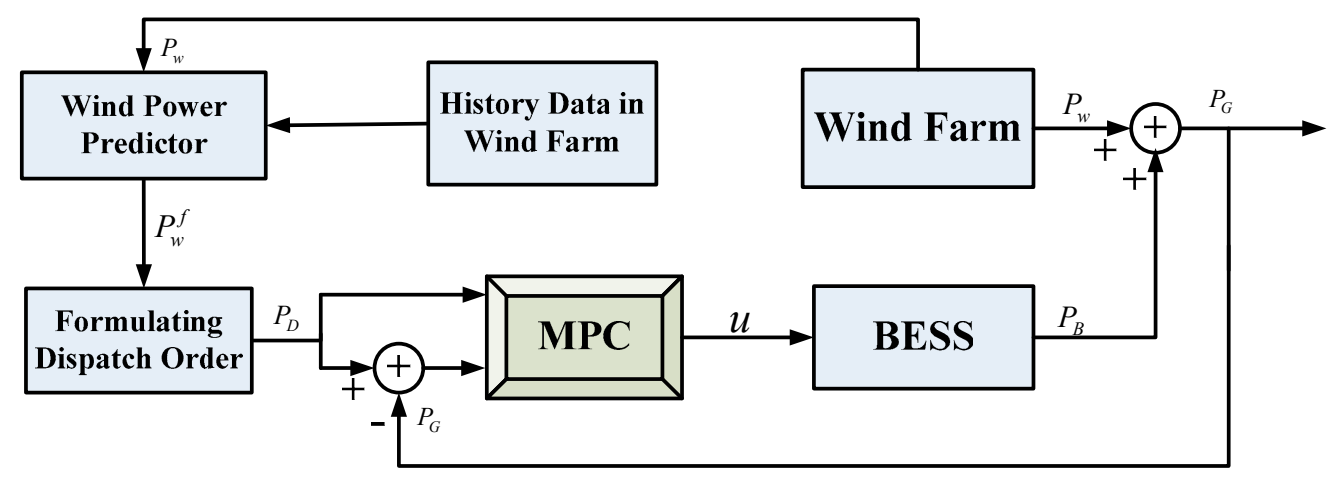

Figure 3. Diagram of smoothing and dispatching wind power via MPC.

The object of the wind/battery energy storage hybrid system is to enable the total output to track the dispatch order extremely closely with the lowest cost of BESS action, i.e., the charging/discharging power of the BESS. In order to achieve this goal to improve the lifespan of the BESS, the function of control performance of the system could be described as the following:

$$
J=\alpha \sum_{i=1}^{m_{p}}\left(P_{\mathrm{G}}(k+i \mid k)-P_{\mathrm{D}}(k+i \mid k)\right)^{2}+(1-\alpha) \sum_{i=1}^{m_{c}-1}(u(k+i \mid k))^{2}
$$

where, $(k+i \mid k)$ is the predicted value based on the information at time $k, m_{p}$ is the predictive horizon, $m_{c}$ is the control horizon, $\alpha \in(0,1)$ is the balance coefficient of the tracing performance and the control action. In order to simplify the calculations, in this paper the predictive horizon equals the control horizon, i.e., $m_{p}=m_{c}=p$.

Together with (2)-(7), the MPC formulation for wind/battery storage hybrid systems used to dispatch short-term wind power can be described as (13):

$$
\begin{gathered}
J=\alpha \sum_{i=1}^{p}\left(y(k+i \mid k)-y_{\text {ref }}(k+i \mid k)\right)^{2}+(1-\alpha) \sum_{i=1}^{p-1}(u(k+i \mid k))^{2} \\
\text { s.t. }\left\{\begin{array}{c}
S_{\min } \leqslant x_{2}(k) / C_{\mathrm{B}} \leqslant S_{\max } \\
P_{c} \leqslant u(k) \leqslant P_{d}
\end{array}\right.
\end{gathered}
$$

where, $y_{\text {ref }}$ is reference trajectory, which in this paper is the dispatch order.

Based on the principle of the MPC, the predicted value $y(k+i \mid k)$ in the predictive horizon could be obtained according to the current system state $x_{\mathrm{i}}(k+1)$ at time $k$ and formulation (2), and it could be described as (15):

$$
\begin{aligned}
y(k+1 \mid k) & =\mathbf{C} x(k+1 \mid k) \\
& =\mathbf{C A} x+\mathbf{C} \mathbf{B}_{1} u(k \mid k)+\mathbf{C B}_{2} d(k) \\
y(k+2 \mid k) & =\mathbf{C} x(k+2 \mid k) \\
& =\left(\mathbf{C A}^{2}+\mathbf{C A}\right) x+\left(\mathbf{C A B}_{\mathbf{1}}+\mathbf{C B}_{\mathbf{1}}\right) u(k \mid k)+\mathbf{C B}_{\mathbf{1}} u(k+1 \mid k)+\left(\mathbf{C A B}_{\mathbf{2}}+\mathbf{C B}_{\mathbf{2}}\right) d(k)+y(k) \\
& \vdots \\
= & \mathbf{C} x(k+p \mid k) \\
= & \sum_{i=1}^{p} \mathbf{C A}^{i} x+\sum_{i=1}^{p} \mathbf{C A}^{i-1} \mathbf{B}_{\mathbf{1}} u(k \mid k)+\sum_{i=1}^{p-1} \mathbf{C A}^{i-1} \mathbf{B}_{1} u(k+1 \mid k)+\cdots \\
& \quad+\sum_{i=1}^{p-m+1} \mathbf{C A}^{i-1} \mathbf{B}_{\mathbf{1}} u(k+m-1 \mid k)+\sum_{i=1}^{p} \mathbf{C A}^{i-1} \mathbf{B}_{\mathbf{2}} d(k)
\end{aligned}
$$

where, $y(k+j \mid k)$ denotes the predicted system response at $k+j$ being calculated at time $k, j=1, \ldots, p$; $u(k+j \mid k)$ denotes the calculated control value at time $k, j=0,1, \ldots, \mathrm{p}-1$; I denotes identity matrix. 
Equation (15) could be described in matrix form:

$$
\mathbf{Y}(k)=\mathbf{G} x(k)+\mathbf{K} \mathbf{U}(k)+\mathbf{L D}(k)
$$

where:

$$
\begin{gathered}
\mathbf{Y}(k)=[y(k+1 \mid k), y(k+2 \mid k), \cdots, y(k+p \mid k)]^{\mathrm{T}} \\
\mathbf{U}(k)=[u(k \mid k), u(k+1 \mid k), \cdots, u(k+p-1 \mid k)]^{\mathrm{T}} \\
\mathbf{D}(k)=[d(k \mid k), d(k+1 \mid k), \cdots, d(k+p-1 \mid k)]^{\mathrm{T}} \\
\quad \mathbf{G}=\left[\mathbf{C A}, \mathbf{C A}^{2}, \cdots, \mathbf{C A}^{p}\right]^{\mathrm{T}}
\end{gathered}
$$

$$
\mathbf{K}=\left[\begin{array}{cccc}
\mathbf{C B}_{1} & \mathbf{0} & \mathbf{L} & \mathbf{0} \\
\mathbf{C A B}_{1} & \mathbf{C} \mathbf{B}_{1} & \mathbf{L} & \mathbf{0} \\
\mathbf{M} & \mathbf{M} & \mathbf{L} & \mathbf{M} \\
\mathbf{C A}^{p-1} \mathbf{B}_{1} & \mathbf{C A}^{p-2} \mathbf{B}_{1} & \mathbf{L} & \mathbf{C} \mathbf{B}_{1}
\end{array}\right]
$$

$$
\mathbf{L}=\left[\begin{array}{cccc}
\mathbf{C B}_{2} & 0 & \cdots & 0 \\
\mathbf{C A B}_{2} & \mathbf{C B}_{2} & \cdots & 0 \\
\vdots & \vdots & \cdots & \vdots \\
\mathbf{C A}^{p-1} \mathbf{B}_{2} & \mathbf{C A}^{p-2} \mathbf{B}_{2} & \cdots & \mathbf{C B}_{2}
\end{array}\right]
$$

Furthermore, in control horizon, indicator function of quadratic performance for controller of the combined system could be described as:

$$
\begin{array}{r}
J=\min (\mathbf{Y}-\mathbf{G} x(k))^{\mathrm{T}} \mathbf{Q}(\mathbf{Y}-\mathbf{G} x(k))-2 \mathbf{U}^{\mathrm{T}} \mathbf{K}^{\mathrm{T}} \mathbf{Q}(\mathbf{Y}-\mathbf{G} x(k))+\mathbf{U}^{\mathrm{T}}\left(\mathbf{K}^{\mathrm{T}} \mathbf{Q K}+\mathbf{R}\right) \mathbf{U} \\
\text { s.t. }\left\{\begin{array}{c}
S_{\min } \leqslant x_{2}(k) / C_{\mathrm{B}} \leqslant S_{\max } \\
P_{c} \leqslant u(k) \leqslant P_{d}
\end{array}\right.
\end{array}
$$

where, $\mathbf{Q}=\left[\begin{array}{cccc}\alpha & 0 & \cdots & 0 \\ 0 & \alpha & \cdots & 0 \\ \vdots & \vdots & \ddots & \vdots \\ 0 & 0 & \cdots & \alpha\end{array}\right], \mathbf{R}=\left[\begin{array}{cccc}1-\alpha & 0 & \cdots & 0 \\ 0 & 1-\alpha & \cdots & 0 \\ \vdots & \vdots & \ddots & \vdots \\ 0 & 0 & \cdots & 1-\alpha\end{array}\right]$.

Equation (13) and the constraints (14) formulate a quadratic programming problem, so rolling optimization is adapted to obtain the optimal control sequence, only the first of which is sent into the system to work. At time $k+1$, the system information updates and the optimal procedure is repeated as above.

\subsection{Rolling Optimization Using Inner Point Method}

In the design for MPC, control sequence $(U)$ is the useful one and the items that have no relationship with $U$ would be neglected, so the control objective would be written as follows:

$$
\mathbf{J}=\mathbf{U}^{\mathrm{T}}\left(\mathbf{\Psi}^{\mathrm{T}} \mathbf{Q} \mathbf{\Psi}+\boldsymbol{R}\right) \mathbf{U}+2\left(\mathbf{\Psi}^{\mathrm{T}} \mathbf{W}\left[\begin{array}{lll}
\mathbf{G} & \mathbf{L} & \mathbf{R}
\end{array}\right]\left[\begin{array}{c}
x \\
d \\
-1
\end{array}\right]\right)^{\mathrm{T}} \mathbf{U}
$$

It is standard form of quadratic programming:

$$
\begin{aligned}
& \min _{x} \frac{1}{2} u^{\mathrm{T}} \mathbf{H} u+f^{\mathrm{T}} u \\
& \text { s.t. } \mathbf{A}_{\text {ineq }} u \leqslant \mathbf{b}_{\text {ineq }}
\end{aligned}
$$


The inner point method is adapted in the paper to solve the control value, the basic line of thought is based on the condition of Karush-Kuhn-Tucker (KKT):

$$
\begin{gathered}
\mathbf{H} u+f+\mathbf{A}_{\text {ineq }}^{\mathrm{T}} z=0 \\
-\mathbf{A}_{\text {ineq }} u+\mathbf{b}_{\text {ineq }}-s=0 \\
s_{i} z_{i}=0, i=1,2, \ldots m \\
s \geqslant 0 ; z \geqslant 0
\end{gathered}
$$

Equation (28) is rewritten in the iteration form:

$$
\beta=\sigma \frac{s^{\mathrm{T}} z}{m}
$$

where, $\sigma$ is the diminish iteration variable converging to $0, \sigma \in(0,1], m$ is the number of inequality constraints. Therefore, the step of inner point to solve the quadratic programming is as follows:

Step 1: Select the initial condition $(x(0), s(0), z(0))$ satisfying $s(0)>0, z(0)>0$.

Step 2: Obtain the $\Delta u$ and $\Delta z$ satisfying the following equation:

$$
\left[\begin{array}{cc}
\mathbf{H} & \mathbf{A}_{\text {ineq }}^{\mathrm{T}} \\
\mathbf{A}_{\text {ineq }} & \boldsymbol{\Gamma}
\end{array}\right]\left[\begin{array}{c}
\Delta u \\
\Delta z
\end{array}\right]=\left[\begin{array}{l}
r_{1} \\
r_{2}
\end{array}\right] \text { and } \Delta s=-s+Z^{-1}(\beta e-T \Delta Z) .
$$

where:

$$
\begin{aligned}
\Gamma=-Z^{-1} S^{k}, S=\left[\begin{array}{cccc}
s_{1} & 0 & \cdots & 0 \\
0 & s_{2} & \cdots & 0 \\
\vdots & \vdots & \ddots & \vdots \\
0 & 0 & \cdots & s_{m}
\end{array}\right], Z=\left[\begin{array}{cccc}
z_{1} & 0 & \cdots & 0 \\
0 & z_{2} & \cdots & 0 \\
\vdots & \vdots & \ddots & \vdots \\
0 & 0 & \cdots & z_{m}
\end{array}\right], e=\left[\begin{array}{c}
1 \\
1 \\
\vdots \\
1
\end{array}\right]_{m_{c} \times 1} \\
r_{1}=-\mathbf{H} u-f-\mathbf{A}_{\text {ineq }}^{-\mathrm{T}} z, r_{2}=-\mathbf{A}_{\text {ineq }} u+b_{\text {ineq }}-\beta \mathbf{Z}^{-1} e .
\end{aligned}
$$

Step 3: Update the variable through increment.

$$
(x(1), s(1), z(1))=(x(0), s(0), z(0))+a(\Delta x(0), \Delta s(0), \Delta z(0)), 0<a<1 .
$$

Step 4: Judge the convergence. If it converges, the procedure would stop and the optimal control values are obtained, otherwise, the value are treated as initial value and sent into step 2 to solve iteratively.

The optimal control sequence is solved by IP for the quadratic programming problem, and only the first of which is sent into the BESS. The next time, the procedure is repeated to realize rolling optimization.

\section{Simulation Results}

In this paper, actual wind power data from a wind farm in northeast China whose capacity is $45 \mathrm{MW}$ and the sampling time interval is $5 \mathrm{~min}$ was adopted. The sodium-sulfur (NaS) battery was selected due to its fast response time. It only takes $10 \mathrm{~s}$ to go from 0 to rated power [27], which means it could operate at rated power in $5 \mathrm{~min}$. The technology for the BESS is max-charging power $P_{\text {max_ch }}$ and max-discharging power $P_{\text {max_f }}$ are both $3 \mathrm{MW}$; initial SOC is 0.5 ; the limit of SOC is $0.2-0.8$.

Data for MPC: the predictive horizon and control horizon should satisfy the relation: $m_{c} \leqslant m_{p}$. In this paper wind power is forecasted $30 \mathrm{~min}$ in the future according to the wind data during the last $12 \mathrm{~h}$, as a consequence, in order to ensure sound control effects, the predictive horizon and control horizon are both set to be $30 \mathrm{~min}$, i.e., $m_{c}=m_{p}=6$, $(30 \mathrm{~min}), \alpha$ is 0.8 . 
In [12], the authors proposed that when the SOC limit is between 0.3 and 0.7 , the capacity of the BESS to dispatch the wind power would be $20 \%-30 \%$ of the rated capacity of the wind farm. As for this paper, the SOC would operate between $0.2-0.8$, therefore, the capacity of the BESS would be lower than that in [12]. In this paper, capacities of $10 \%$ and $20 \%$ would be selected to test the proposed strategy. Based on the predicted wind power using the ARIM model developed in [26] and the method in Section 2.1, the dispatch curve in a day was acquired and illustrated in Figure 4.

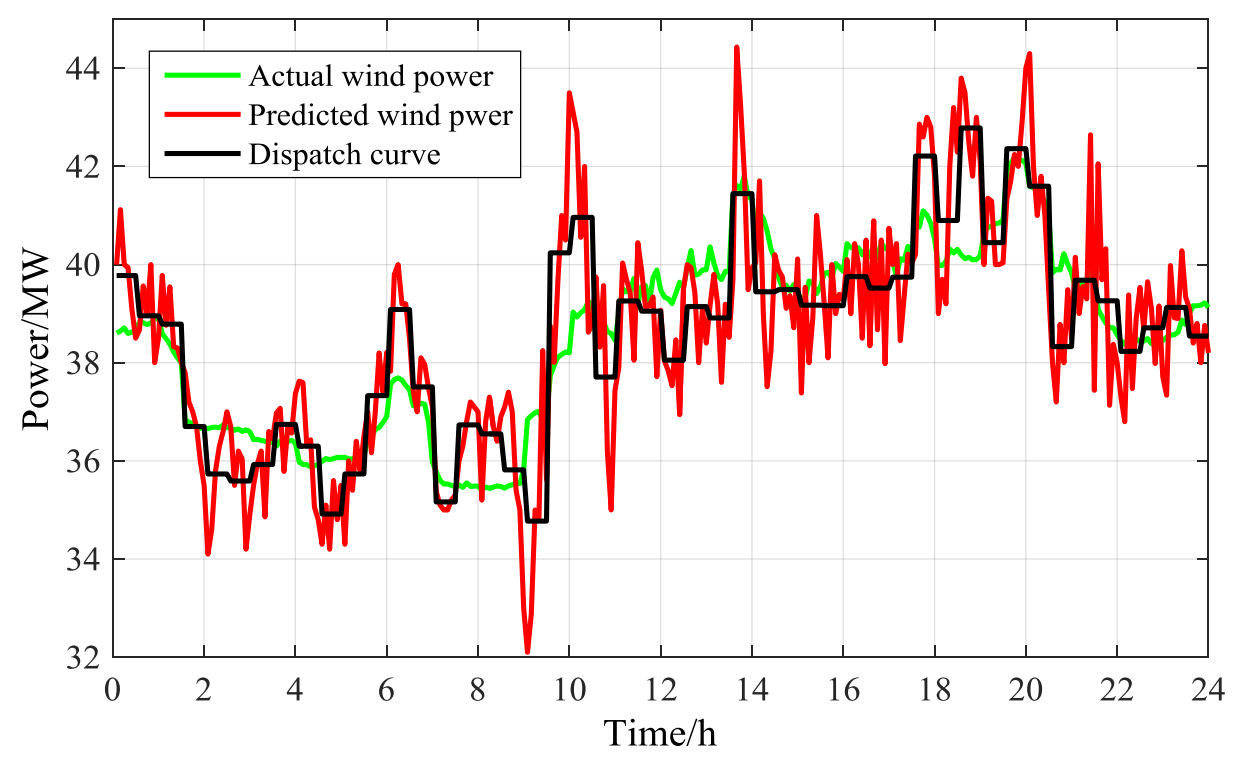

Figure 4. Wind power and dispatching curve.

\subsection{Capacity of BESS in $4.5 \mathrm{MWh}$}

The dispatch strategy basing on MPC in Section 3 is applied to dispatch a wind farm whose rated capacity is $45 \mathrm{MW}$ with a BESS of $45 \mathrm{MWh}$ rate capacity, to maintain the total output from the wind/battery storage hybrid system to effectively track the dispatch order shown in Figure 4. Meanwhile, the effects of the proposed strategy are compared with those of a traditional solution for MPC, the QP method, which is illustrated in Figure 5.

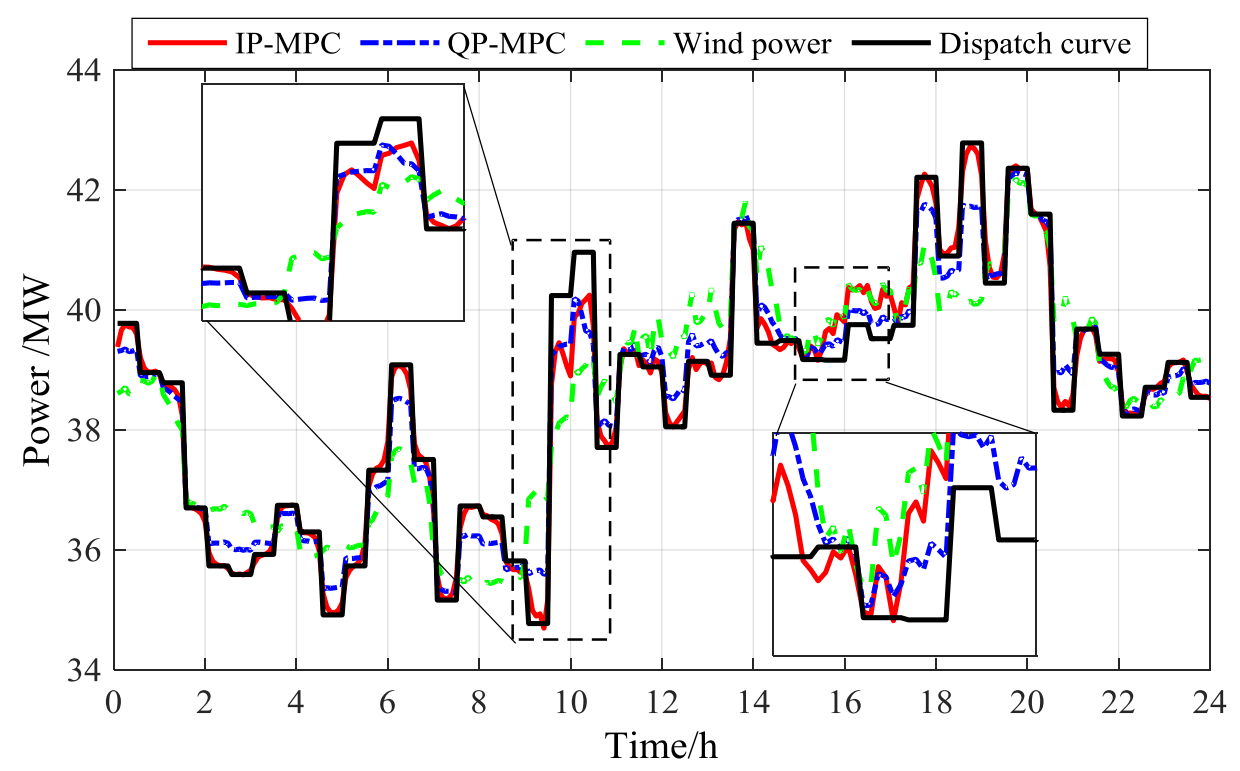

Figure 5. Wind power and dispatch power (4.5 MWh). 
In Figure 5 for both methods, the effects of the tracing are mostly nice. The IP method has a better performance on the whole even though there would be some worse tracing performance between 15 and $17 \mathrm{~h}$.

The statistical analysis on the absolute deviation between diapatched power and dispatch order in Figure 5 is illustrated in Table 1 and the statistical result of relative deviation is shown in Figure 6.
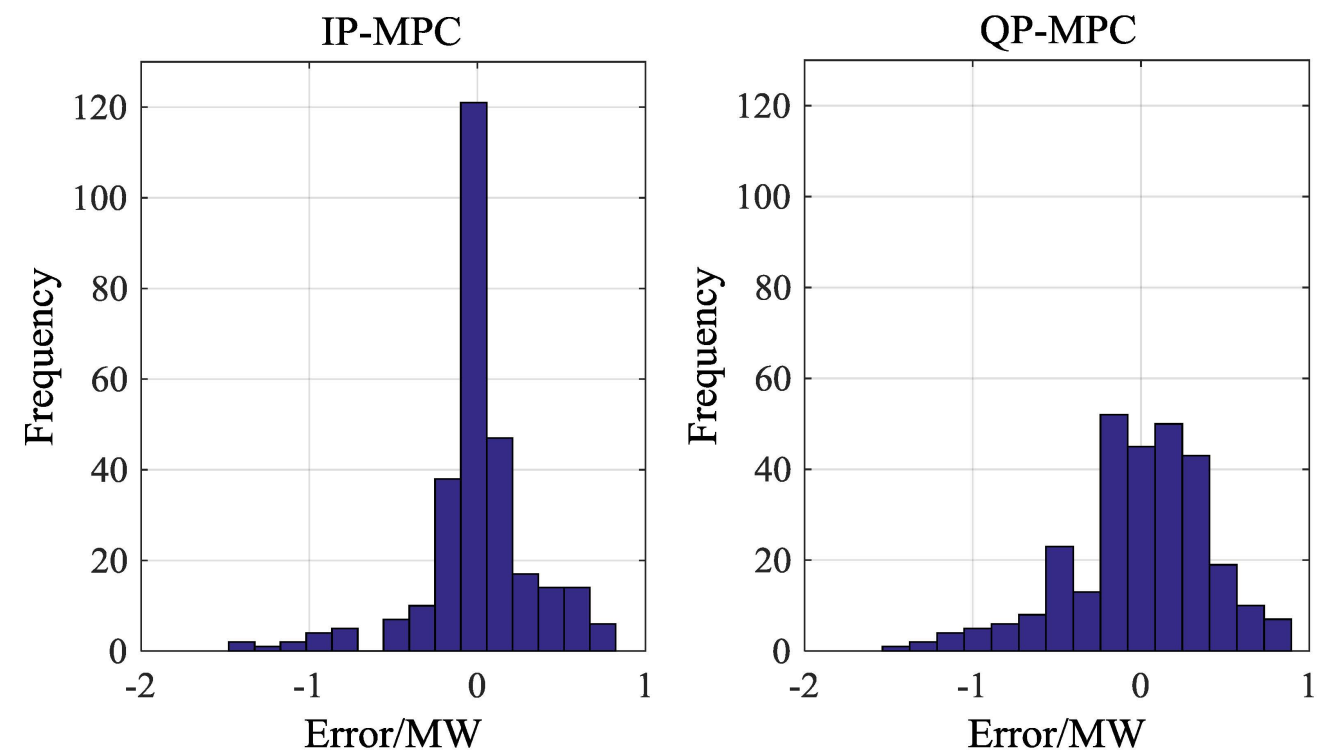

Figure 6. Frequency distribution of dispatching error (4.5 MWh).

Table 1. Statistic result of dispatching error (4.5 MWh).

\begin{tabular}{|c|c|c|c|c|c|}
\hline \multirow{2}{*}{ Method } & \multicolumn{2}{|c|}{ Max Deviation } & \multirow{2}{*}{$\begin{array}{l}\text { Min Deviation } \\
\text { (MW) }\end{array}$} & \multirow{2}{*}{$\begin{array}{l}\text { Mean } \\
\text { (MW) }\end{array}$} & \multirow{2}{*}{$\begin{array}{l}\text { Std. } \\
\text { (MW) }\end{array}$} \\
\hline & Value (MW) & Time & & & \\
\hline IN-MPC & 1.478 & 10 & 0 & 0.2047 & 0.1096 \\
\hline QP-MPC & 1.537 & 13 & 0 & 0.4139 & 0.1706 \\
\hline
\end{tabular}

As can be shown in Table 1, for the IN-MPC strategy proposed in this paper, the maximum deviation is $1.478 \mathrm{MW}$, however, for the QP-MPC, it is $1.537 \mathrm{MW}$, meanwhile, the mean value of the proposed one is $0.2047 \mathrm{MW}$ which is smaller than the $0.4139 \mathrm{MW}$ solved by the conventional one. Therefore, the dispatching results based on IP-MPC are better due to its algorithm being better than QP-MPC. The results can also be verified in Figure 6, where as can be seen, the frequency of smaller errors in IP-MPC would be less than that in QP-MPC.

Figures 7 and 8 explain the reason for the "bad points". In Figure 6 both curves are strictly limited between 0.2 and 0.8 , during $15-17 \mathrm{~h}$, SOC for IP-MPC almost reaches the upper limits, therefore the BESS has no space for BESS to absorb energy. Furthermore, in Figure 8, the action of BESS is little and less than that in QP-MPC. The operating range of the BESS is limited within $[-3,3]$, most of which are in half the limited power region of $[-1.5,1.5]$. As a consequence, capacity is an important factor in the performance. In the next part, larger capacity would be selected. 


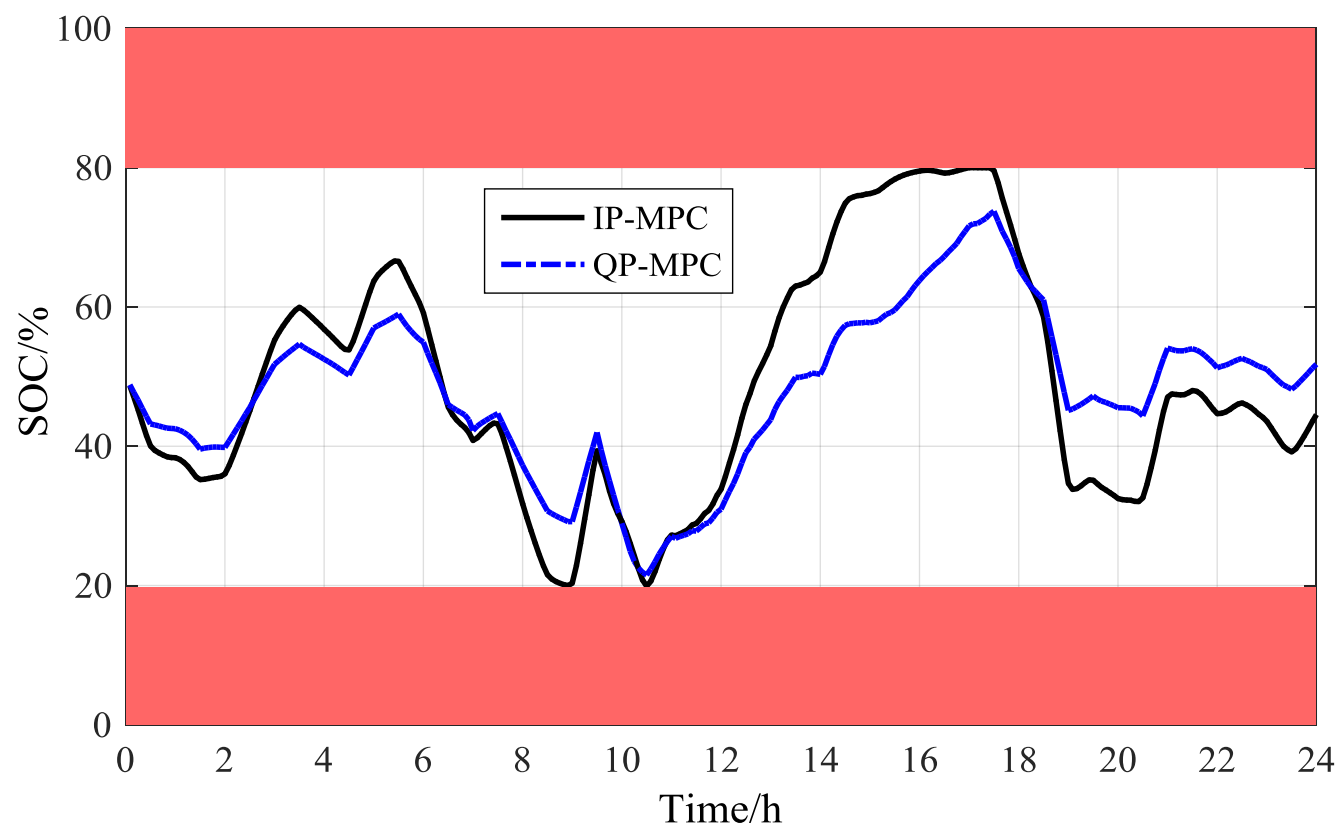

Figure 7. SOC of BESS (4.5 MWh).

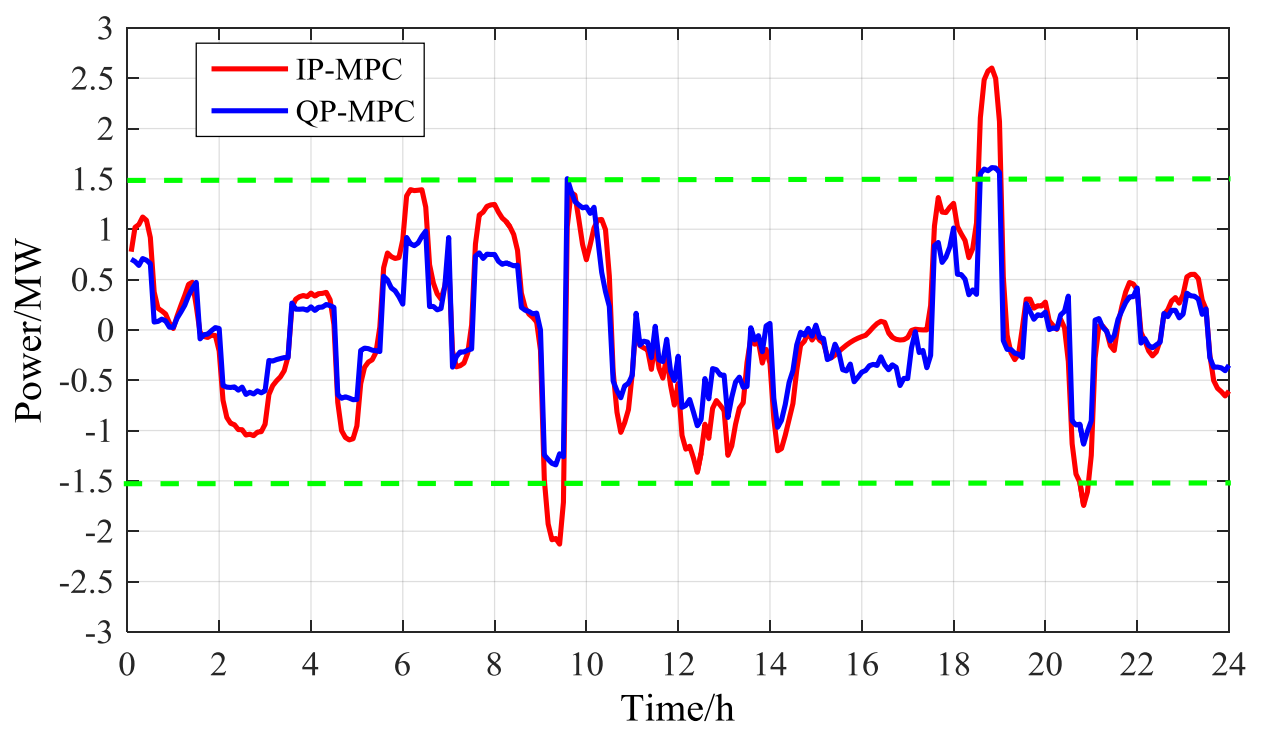

Figure 8. Power response of BESS (4.5 MWh).

\subsection{Capacity of BESS in $9 \mathrm{MWh}$}

In this section, the capacity of the BESS is doubled, which is $20 \%$ rated capacity of the wind farm, i.e., 9 MWh. The proposed strategy is applied to dispatch and control active power from the wind/battery storage hybrid system for one day $(24 \mathrm{~h})$, and the results are shown in Figure 9. In Figure 9, both have a better performance than that in Figure 5 because of the larger capacity of the BESS. The total output under the control of IP could trace the dispatch order perfectly, and the performance of IP-MPC is better than that of QP-MPC.

The statistical analysis on both absolute deviation and relative deviation from the dispatching order is shown in Table 2 and Figure 10. The comparison between Tables 1 and 2 shows that due to the larger capacity of the BESS, the maximum deviation is only MW with the proposed strategy, and the tracking performance is better than that with 4.5 MWh. 


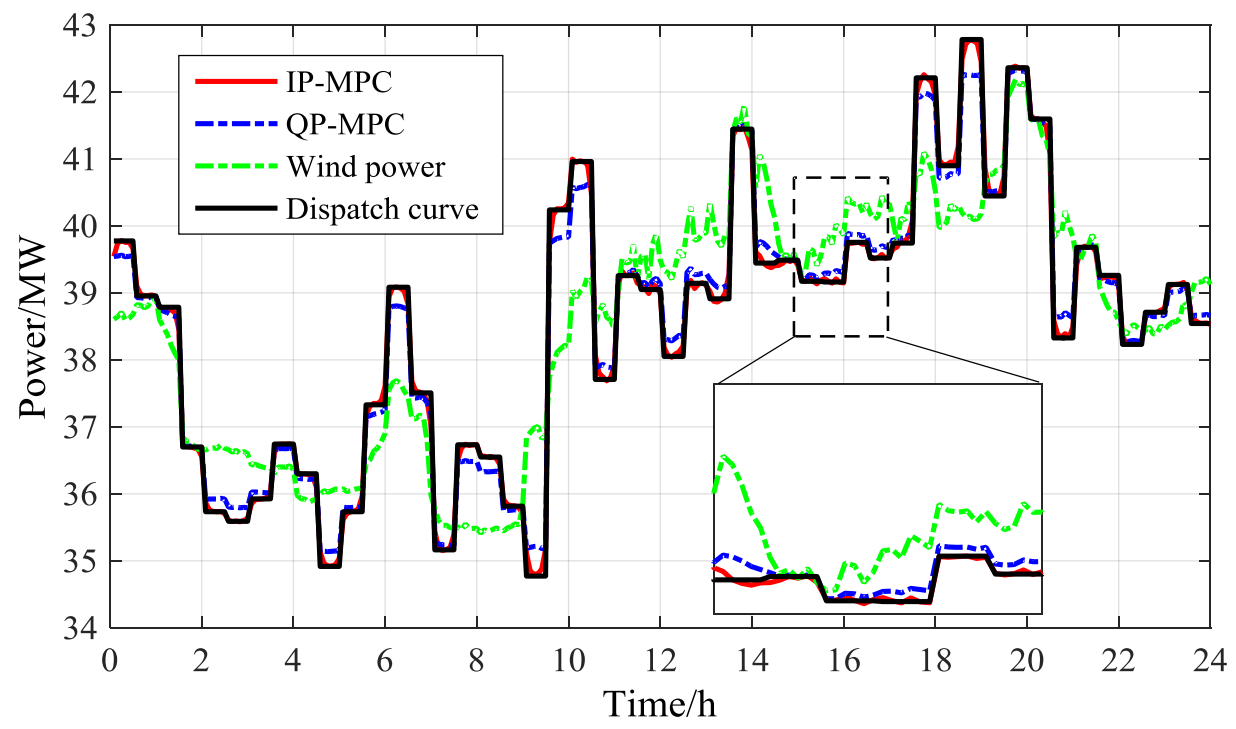

Figure 9. Wind power and dispatch power (9 MWh).
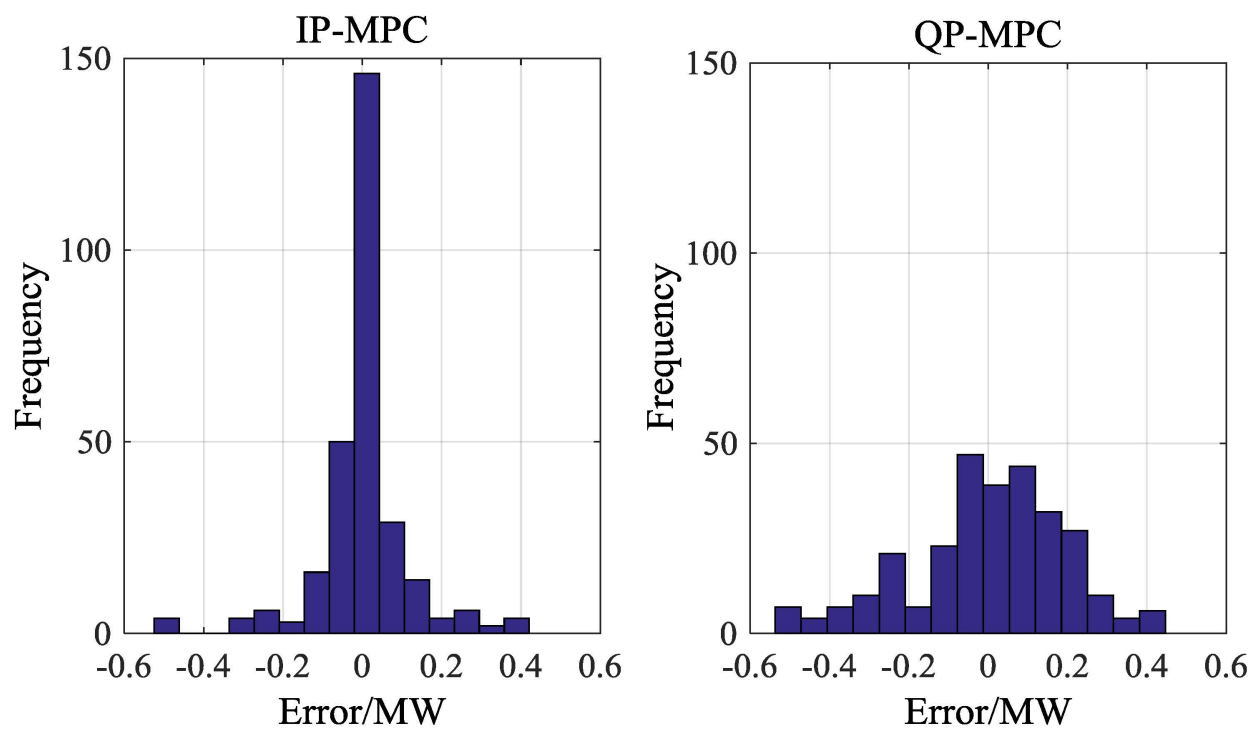

Figure 10. Frequency distribution of dispatching error (9 MWh).

Table 2. Statistic result of dispatching error (9 MWh).

\begin{tabular}{cccccc}
\hline \multirow{2}{*}{ Method } & \multicolumn{2}{c}{ Max Deviation } & \multirow{2}{*}{$\begin{array}{c}\text { Min Deviation } \\
\text { (MW) }\end{array}$} & $\begin{array}{c}\text { Mean } \\
\text { (MW) }\end{array}$ & $\begin{array}{c}\text { Std. } \\
\text { (MW) }\end{array}$ \\
\cline { 2 - 3 } Value (MW) & Time & & & & \\
\hline IN-MPC & 0.524 & 21 & 0 & 0.0689 & 0.0142 \\
QP-MPC & 0.537 & 19 & 0 & 0.1529 & 0.0385 \\
\hline
\end{tabular}

As for errors in Figure 12, IP-MPC is less error prone than QP-MPC. Figure 11 explains the effects in Figure 9, where neither has reached the limiting bounds of 0.2 and 0.8 , which means the BESS has sufficient energy to charge and discharge power to maintain an excellent performance. In Figure 12, the action of the BESS operates in the limited range, and most time, both run between -1.5 and 1.5. 


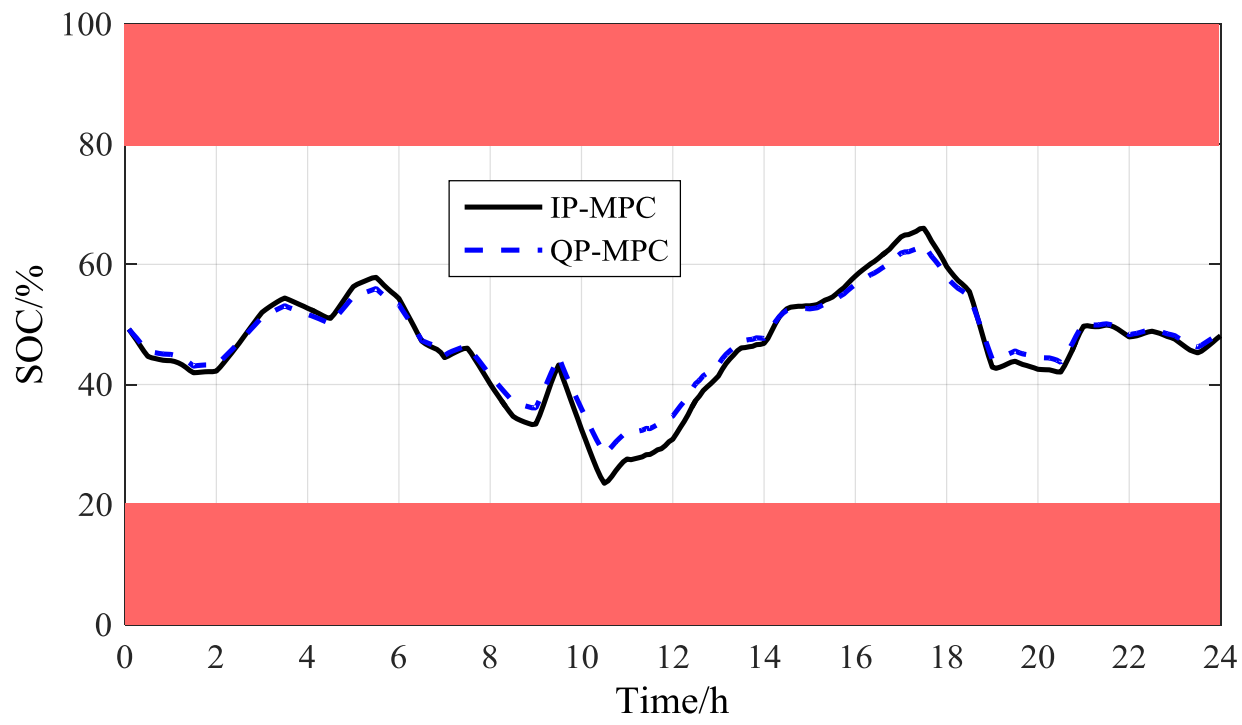

Figure 11. SOC of BESS (9 MWh).

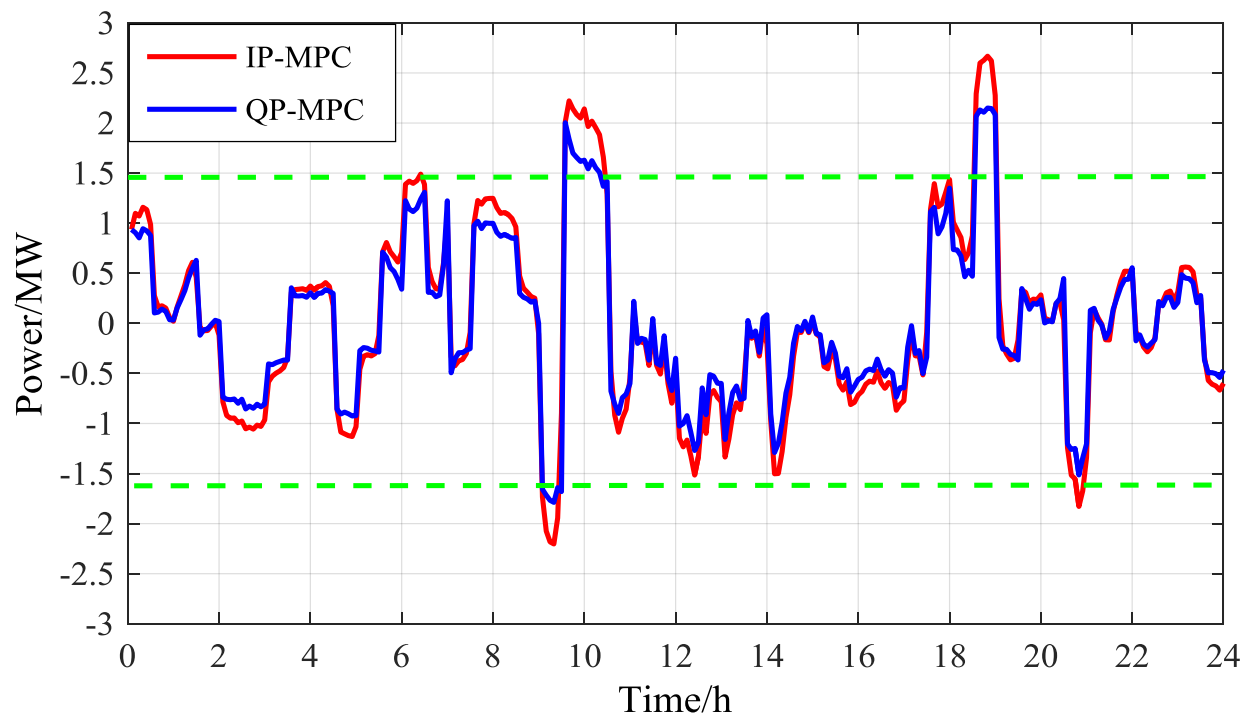

Figure 12. Power response of BESS (9 MWh).

From both simulations, when the capacity of the BESS is small, IP-MPC has a better performance in tracking dispatch orders8 than QP-MPC. When the capacity is bigger, the tracking results of both are almost the same. Due to the high cost of the BESS with big capacity, IP-MPC has an edge in the market.

\section{Conclusions}

This paper developed a MPC-based dispatching strategy for wind/battery energy storage hybrid systems. Firstly, the dispatch curve in the dispatching interval is obtained using the predicted wind power, and then the MPC controller calculates the control value for the BESS. The simulation results show that: (a) the proposed strategy based on MPC could enable the active output of a wind/battery energy storage hybrid system to trace the dispatch order, which improves the schedulablity of the wind farm; (b) the IP-MPC has a better performance in tracking dispatch orders than QP-MPC under the same BESS conditions, such as capacity, limited SOC and charging/discharging power limits. This paper focuses on the strategy for BESS based on MPC to smooth and dispatch wind power. Our future work will investigate the errors in wind power prediction and the stochastic nature of wind power. 
Acknowledgments: The authors gratefully acknowledge the support of the National Natural Science Foundation of China (No.51507028 and No.51377017), the national key research and development plan (2016YFB0900100) and the Hong Kong Polytechnic University under Project G-UA3Z.

Author Contributions: Deyou Yang and Guowei Cai conceived and designed the experiments; Jiaxin Wen performed the experiments; Jiaxin Wen and Ka-wing Chan analyzed the data, Deyou Yang and Jiaxin Wen wrote this paper.

Conflicts of Interest: The authors declare no conflict of interest.

\section{Nomenclature}

\begin{tabular}{|c|c|}
\hline Symbol & Description \\
\hline$P_{\mathrm{G}} / x_{1}$ & Total output of the wind/battery energy storage hybrid system \\
\hline$P_{\mathrm{B}} / u$ & The action of the BESS/control value \\
\hline$P_{\mathrm{W}} / d$ & Actual wind power/disturbance \\
\hline$E_{\mathrm{B}} / x_{2}$ & The remaining energy in a BESS \\
\hline SOC & State of Charge of a BESS \\
\hline$\Delta T_{\mathrm{B}}$ & Sampling time \\
\hline$C_{\mathrm{B}}$ & Rate capacity of a BESS \\
\hline$S_{\min }$ & Lower limits of a BESS \\
\hline$S_{\max }$ & Upper limit of a BESS \\
\hline$P_{\text {max_ch }}$ & Maximum of charging power of a BESS \\
\hline$P_{\text {max_f }}$ & Maximum of discharging power of a BESS \\
\hline$P_{c}$ & Maximum available charging power of a BESS \\
\hline$P_{d}$ & Maximum available discharging power of a BESS \\
\hline$a_{t}$ & White noise time series \\
\hline$z_{t}$ & Time series \\
\hline$\varphi, \theta$ & Constants in ARMA \\
\hline$F$ & Backward difference operator \\
\hline$T_{d}$ & Dispathcing interval \\
\hline$P_{\mathrm{D}} / y_{\text {ref }}$ & Dispatching power \\
\hline$\alpha$ & Balance coefficient of the tracking performance and control action \\
\hline$m_{p} / p$ & Prediction horizon \\
\hline$m_{c}$ & Control horizon \\
\hline$y$ & Predicted system response \\
\hline $\mathbf{A} / \mathbf{B}_{1} / \mathbf{B}_{2} / \mathbf{C}$ & Model parameter for expression (2) \\
\hline $\mathbf{Y}$ & Predicted output in matrix form \\
\hline $\mathbf{U}$ & Control value in matrix form \\
\hline D & Disturbance in matrix form \\
\hline $\mathrm{G} / \mathrm{K} / \mathrm{L} / \Psi / \mathrm{W}$ & The matrices after being processed \\
\hline $\mathrm{Q} / \mathrm{R}$ & Weight matrices \\
\hline $\mathbf{H} / f$ & Matrices in standardquadratic programming \\
\hline $\mathbf{A}_{\text {ineq }}$ & Liner inequality matrix \\
\hline $\mathbf{b}_{\text {ineq }}$ & Liner inequality vetor \\
\hline$\beta$ & Interation variable \\
\hline$\sigma$ & Diminish interation variable \\
\hline$s$ & Slack variable \\
\hline$z$ & Lagrange multipliers corresponding to s \\
\hline$m$ & The number of inequality constraints \\
\hline$r_{1}$ & Dual residual \\
\hline$r_{2}$ & Inequality constraint residual \\
\hline$a$ & Iterative coefficient \\
\hline
\end{tabular}




\section{References}

1. The World Sets New Wind Installations Record: 63,7 GW New Capacity In 2015. Available online: http: / /www.wwindea.org/the-world-sets-new-wind-installations-record-637-gw-new-capacity-in-2015/ (accessed on 9 August 2016).

2. Fox, B. Introduction. In Wind Power Integration: Connection and System Operational Aspects; IET Power and Energy Series: Stevenage, UK, 2007; Volume 50, pp. 77-85.

3. Bouffard, F.; Galiana, F.D. Stochastic security for operation planning with significant wind power generation. IEEE Trans. Power Syst. 2007, 23, 306-316. [CrossRef]

4. Liu, J.Z. Basic issues of utilization of large-scale renewable power with high security and efficiency. Proc. CSEE 2013, 33, 1-9.

5. Barton, J.P.; Infield, D.G. Energy storage and its use with intermittent renewable energy. IEEE Trans. Energy Convers. 2004, 19, 441-448. [CrossRef]

6. Divya, K.C.; Østergaard, J. Battery energy storage technologies for power system-An overview. Electr. Power Syst. Res. 2008, 79, 511-520. [CrossRef]

7. $\mathrm{Ru}, \mathrm{Y}$;; Kleissl, J.; Martinez, S. Storage size determination for grid-connected photovoltaic systems. IEEE Trans. Sustain. Energy 2013, 4, 68-81. [CrossRef]

8. Bindner, H.; Lundsager, P. Integration of wind power in the power system. In Proceedings of the 28th Annual Conference of the Industrial Electronics Society, Sevilla, Spain, 5-8 November 2002.

9. Teleke, S.; Baran, M.E.; Bhattacharya, S. Optimal Control of Battery Energy Storage for Wind Farm Dispatching. IEEE Trans. Energy Convers. 2010, 25, 787-794. [CrossRef]

10. Jia, H.X.; Zhang, Y.; Wang, Y.F. Application of Energy storage technology in wind power systems. Renew. Energy 2009, 27, 10-15.

11. Nguyen, T.T.; Yoo, H.J.; Kim, H.M. A Flywheel Energy Storage System Based on a Doubly Fed Induction Machine and Battery for Microgrid Control. Energies 2015, 8, 5074-5089. [CrossRef]

12. Teleke, S.; Baran, M.E.; Huang, A. Control strategies for battery storage for wind farm dispatching. IEEE Trans. Energy Convers. 2009, 24, 725-732. [CrossRef]

13. Wang, X.Y.; Vilathgamuwa, D.M.; Choi, S.S. Determination of battery storage capacity in energy buffer for wind farm. IEEE Trans. Energy Convers. 2008, 23, 868-878. [CrossRef]

14. Jiang, Q.; Wang, H. Two-time-scale coordination control for a battery energy storage system to mitigate wind power fluctuations. IEEE Trans. Energy Convers. 2013, 28, 52-61. [CrossRef]

15. Nguyen, C.L.; Lee, H.H.; Chun, T.W. Cost-optimized battery capacity and short-term power dispatch control for wind farm. IEEE Trans. Ind. Appl. 2015, 51, 595-606. [CrossRef]

16. Arulampalam, A.; Barnes, M.; Jenkins, N. Power quality and stability improvement of a wind farm using STATCOM supported with hybrid battery energy storage. IEE Proc.-Gener. Transm. Distrib. 2006, 153, 701-710. [CrossRef]

17. Zeng, J.; Zhang, B.; Mao, C. Use of battery energy storage system to improve the power quality and stability of wind farms. In Proceedings of the 26th International Conference on Power system Technology, Chongqing, China, 22-26 October 2006.

18. Trung, T.T.; Ahn, S.J.; Choi, J.H. Real-Time Wavelet-Based Coordinated Control of Hybrid Energy Storage Systems for Denoising and Flattening Wind Power Output. Energies 2014, 7, 6620-6644. [CrossRef]

19. Yoshimoto, K.; Nanahara, T.; Koshimizu, G. New control method for regulating state-of-charge of a battery in hybrid wind power/battery energy storage system. In Proceedings of the Power System Conference and Exposition, Atlanta, GA, USA, 29 October-1 November 2006.

20. Hong, H.S.; Jiang, Q.Y.; Yan, Y.T. An optimization control method of battery smoothed in real time. Autom. Electr. Power Syst. 2012, 37, 103-109.

21. Vahidi, A.; Stefanopoulou, A.; Peng, H. Current management in a hybrid fuel cell power system: A model-predictive control approach. IEEE Trans. Control Syst. Technol. 2006, 14, 1047-1057. [CrossRef]

22. Kassem, A.M.; Yousef, A.M. Voltage and frequency control of an autonomous hybrid generation system based on linear model predictive control. Sustain. Energy Technol. Assess. 2013, 4, 52-61.

23. Torreglosa, J.P.; García, P.; Fernández, L.M.; Jurado, F. Energy dispatching based on predictive controller of an off-grid wind turbine/photovoltaic/hydrogen/battery hybrid system. Renew. Energy 2015, 74, 326-336. [CrossRef] 
24. Wu, W.; Xu, J.P.; Hwang, J.J. Multi-loop nonlinear predictive control scheme for a simplistic hybrid energy system. Int. J. Hydrog. Energy, 2009, 34, 3953-3964. [CrossRef]

25. Kou, P.; Gao, F.; Guan, X.H. Stochastic predictive control of battery energy storage for wind farm dispatching: Using probabilistic wind power forecasts. Renew. Energy 2015, 80, 286-300. [CrossRef]

26. Roy, B.; Bipul, K.; Rajesh, K. Unit Commitment Risk Analysis of Wind Integrated Power Systems. IEEE Trans. Power Syst. 2009, 24, 930-939.

27. Sodium Sulfur Battery Catalog; NGK Insulators Ltd.: Tokyo, Japan, 2009.

(c) 2016 by the authors; licensee MDPI, Basel, Switzerland. This article is an open access article distributed under the terms and conditions of the Creative Commons Attribution (CC-BY) license (http://creativecommons.org/licenses/by/4.0/). 